Canadian

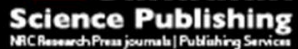

Canadian Journal of Physiology and Pharmacology Revue canadienne de physiologie et pharmacologie

\title{
Selective inhibition of HDAC2 by magnesium valproate attenuates cardiac hypertrophy
}

\begin{tabular}{|r|l|}
\hline Journal: & Canadian Journal of Physiology and Pharmacology \\
\hline Manuscript ID & cjpp-2016-0542.R1 \\
\hline Danuscript Type: & Article \\
\hline Complete List of Authors: & $\begin{array}{l}\text { Raghunathan, Suchi; Institute of Pharmacy, Nirma University } \\
\text { Goyal, Ramesh; Delhi Pharmaceutical Sciences Research University } \\
\text { Patel, Bhoomika; Institute of Pharmacy, Nirma University, Pharmacology }\end{array}$ \\
\hline Keyword: & $\begin{array}{l}\text { Class I HDAC, Class II HDAC, Magnesium Valproate, Partial abdominal } \\
\text { aortic constriction (PAAC) }\end{array}$ \\
\hline \multicolumn{2}{|c}{} \\
\hline \multicolumn{2}{|c}{} \\
\hline
\end{tabular}


Selective inhibition of HDAC2 by magnesium valproate attenuates cardiac hypertrophy

\author{
Suchi Raghunathan ${ }^{1}$, Ramesh K. Goyal ${ }^{2}$, Bhoomika M. Patel ${ }^{1}$ \\ ${ }^{1}$ Institute of Pharmacy, Nirma University, Ahmedabad 382 481, India \\ ${ }^{2}$ Delhi Pharmaceutical Sciences Research University, Delhi, India
}

* Address for Correspondence:

Dr. Bhoomika M. Patel

Assistant Professor

Institute of Pharmacy

Nirma University

Sarkhej-Gandhinagar Highway,

Ahmedabad 382481

Gujarat, India

Phone No: +91 2717 241900-04

Fax No: +91 2717241916

Email: drbhoomikampatel@gmail.com 


\section{ABSTRACT}

The regulatory paradigm in cardiac hypertrophy involves alterations in gene expression that is mediated by chromatin remodeling. Various data suggest that class I and class II histone deacetylases (HDACs) play opposing roles in the regulation of hypertrophic pathways. To address this, we tested the effect of magnesium valproate $(\mathrm{MgV})$, an HDAC inhibitor with 5 times more potency on Class I HDAC. Cardiac hypertrophy was induced by Partial abdominal aortic constriction in wistar rats, and at end of 6 weeks, we evaluated hypertrophic, hemodynamic and oxidative stress parameters, and mitochondrial DNA concentration. Treatment with $\mathrm{MgV}$ prevented cardiac hypertrophy, improved hemodynamic functions, prevented oxidative stress and increased mitochondrial DNA concentration. $\mathrm{MgV}$ treatment also increased the survival rate of the animals as depicted by Kaplan-Meier curve. Improvement in hypertrophy due to HDAC inhibition was further confirmed by HDAC mRNA expression studies which revealed that $\mathrm{MgV}$ decreases expression of pro-hypertrophic HDAC i.e. HDAC2 without altering the expression of anti-hypertrophic HDAC5. Selective class I HDAC inhibition is required for controlling cardiac hypertrophy. Newer HDAC inhibitors which are class I inhibitor and class II promoter can be designed to obtain a 'pan' or 'dual' natural HDAC 'regulators'.

Key Words: Class I HDAC, Class II HDAC, Magnesium Valproate, Partial abdominal aortic constriction (PAAC) 


\section{INTRODUCTION}

Cardiac hypertrophy is a compensatory and adaptive response to a variety of physiological or pathological stimuli like pressure or volume stress, infarction induced loss of contractile mass and sarcomeric protein mutations (Frey et al 2004). Currently there is no specific treatment for cardiac hypertrophy which is associated with several complications including diabetes, hypertension and atherosclerosis (Goyal and Mehta 2013; Patel and Mehta 2012, Patel and Mehta 2013; Raghunathan and Patel 2013). Hence, identification of lead targets for cardiac hypertrophy is the need of hour.

The regulatory paradigm in cardiac hypertrophy involves alterations in gene expression that is mediated by chromatin remodeling. Histone acetylation and deacetylation is regulated by two sets of opposing enzymes- histone acetyltransferases (HATs) and histone deacetyalases (HDACs) respectively. HDACs, remove acetyl group from histones, resulting in its hypoacetylation which diminishes the accessibility for transcription factors to bind to DNA, leading to repression of transcription (de Ruijter et al 2003). HDACs are composed of a highly conserved family, that fall into three subfamilies (de Ruijter et al 2003). The Class I HDAC enzymes which are primarily located in the nucleus consist of $\operatorname{HDAC} 1,2,3$ and 8 (Gregoretti et al 2004). On the other contrary, class II HDAC enzymes shuttle between the cytoplasm and nucleus. Class II HDACs are further classified into class IIa (HDAc 4, 5, 7 and 9) and class IIb (HDAC 6 \& 10) (de Ruijter et al 2003). HDAC 11, which is zinc dependent, shares similarity to class I and II HDAC enzymes and hence is uncategorized (de Ruijter et al 2003).

Various data suggest that class I and class II HDACs play opposing roles in the regulation of hypertrophic pathways. Kee et al (2006) reported predominant involvement of class I HDACs in hypertrophic response in hypertrophic animal models. Nuclear localization of class II HDACs, and thus their ability to repress Myocyte enhance factor-2 (Mef2) dependent hypertrophic gene expression, is regulated by phosphorylation of critical serine residues that 
are conserved in class II, but not class I, HDACs (Chang et al 2004). Thus, class I HDAC and class II HDAC play opposing roles in regulation of cardiac hypertrophy. However, nonspecific HDAC inhibitors (HDACi) like Trichostatin A (TSA) has been found to regress hypertrophy (Kook et al 2003). To address this, we have carried out mRNA expression studies of selected HDACs of both class I and class II HDACs in a pathological model of hypertrophy. Since there are no clinically available specific class I and class II HDAC inhibitors, we have used magnesium valproate as a model drug which is an HDACi with 5 times more sensitivity to class I HDAC (de Ruijter et al 2003). Moreover, Magnesium valproate has been reported to exhibit beneficial effect on cardiovascular complications associated with type 2 diabetes mellitus (Patel et al 2014).

\section{METHODS}

The protocol of the experiment was approved by our institutional animal ethics committee as per the guidance of Committee for the Purpose of Control and Supervision of Experiments on Animals (CPCSEA), Ministry of social justice and Empowerment, Government of India (Protocol No: IPS/PCOL/MPH12-13/1017, dated 16 August 2012).

\subsection{Animals and Chemicals}

Wistar rats of either sex at 6-8 week of age $(n=40)$, weighing 250-350 gms were chosen for the study and maintained under well-controlled conditions of temperature $\left(22 \pm 2^{\circ} \mathrm{C}\right)$, humidity $(55 \pm 5 \%)$ and $12 \mathrm{~h} / 12 \mathrm{~h}$ light-dark cycle. Standard laboratory rat chow and UVfiltered water was provided ad libitum. Magnesium Valproate was received as a gratis supply from ROAQ Chemicals Pvt. Ltd., Vadodara, Gujarat, India. Isoproterenol, Bis-Benzimide H33258, Deoxyribonucleic Acid Sodium Salt were procured from MP Biomedicals India Pvt.

Ltd. RNAse A was received as a gratis supply from Roche Diagnostics India Pvt. Ltd., Mumbai. All other chemicals used in the experiment were of analytical grade.

\subsection{Partial Abdominal Aortic Constriction (PAAC)}


Randomization was carried out and the animals were divided into following groups: control $(\mathrm{CON})$, control treated with magnesium valproate (COM), hypertrophic control (DIS) and hypertrophic treated with magnesium valproate (DIM). Treatment of magnesium valproate was started from $0^{\text {th }}$ day in sham treated and PAAC treated animals. Surgical procedure was done on 3rd day in PAAC control and PAAC treated animal under anesthesia. Incision was made in abdominal wall to expose abdominal aorta. Abdominal aorta was ligated suprarenally with 4.0 silk suture along with $7-0 \mathrm{~mm}$ blunt needle. Thereafter, needle was removed to leave abdominal aorta partially constricted. Sham control and Sham treated animal underwent the same surgical procedure without constricting the abdominal aorta (Patel and Desai 2014). All surgical procedures and hemodynamic assessment were performed with the animals under anesthesia with ketamine $(20 \mathrm{mg} / \mathrm{kg} \mathrm{IM})$ and xylazine (10 $\mathrm{mg} / \mathrm{kg} \mathrm{IM).} \mathrm{Magnesium} \mathrm{valproate} \mathrm{was} \mathrm{given} \mathrm{by} \mathrm{per} \mathrm{oral} \mathrm{route} \mathrm{in} \mathrm{the} \mathrm{dose} \mathrm{of} 210 \mathrm{mg} / \mathrm{kg} / \mathrm{day}$ (by converting human dose to animal dose). The treatment was given in group 3 and 4 for 6 weeks during which body weight changes and mortality rate was monitored.

\subsection{Blood Sample Collection and Serum Analysis}

At the end of five weeks, blood samples were collected from the retro orbital plexuses of each rat under light ether anesthesia. Serum was separated and were analyzed for triglycerides, total cholesterol, very low density lipoprotein (VLDL), low density lipoprotein (LDL), high density lipoprotein (HDL), C-Reactive Protein (CRP), Lactate Dehydrogenase (LDH) and Creatinine Kinase-MB (CK-MB) through biochemical analyzer (Prietest TOUCH Biochemistry Analyzer, 2.622 A, ROBONIK Pvt. Ltd.) using available biochemical diagnostic kits (Labcare Diagnostics Pvt. Ltd., India) (Goyal et al. 2008; Goyal et al 2009).

\subsection{Measurement of Hemodynamic Parameters}

After the study period of 6 weeks, the assessment of hemodyanamic parameters was carried out using invasive cannulation method (Goyal et al. 2011, Patel et al. 2013). In brief, the animals were anaesthetized using $20 \mathrm{mg} / \mathrm{kg}$ ketamine and $10 \mathrm{mg} / \mathrm{kg}$ xylazine, both being 
administered by i.m. route, individually. The optimum body temperature of $37 \pm 1{ }^{\circ} \mathrm{C}$ was maintained throughout the measurement. The upper abdomen was opened and trachea was located and the carotid artery behind it was cannulated. The hemodynamics were measure using BP 100 transducer (Labscrib Systems, IWORX, New Hampshire, USA). All the data were analyzed using Labscribe software (Version 118) (Raghunathan et al. 2014).

\subsection{Measurement of Hypertrophic Parameters}

Once the hemodynamics were measure in the rats, they were euthanized by using an overdose of ketamine $(80 \mathrm{mg} / \mathrm{kg} \mathrm{IM})$. The hearts were removed and other extraneous tissues were discarded. The wet weight of entire heart, left ventricle and right ventricle were recorded and femur length was noted down to calculate various hypertrophic indices like cardiac hypertrophic index (CHI) and left ventricular (LV) hypertrophy index (LVHI) as per previously reported method (Goyal et al. 2011, Patel and Bhadada 2014). Using screw gauge micrometer LV wall thickness was also noted down (Rayabarapu and Patel 2014).

\subsection{Oxidative stress measurement}

Oxidative stress levels were measured in the left ventricular tissue of heart samples by preparing homogenate of left ventricle (Goyal and Mehta 2012; Patel et al. 2012). Quantification of tissue protein levels (Lowry et al. 1951), malondialdehyde levels (MDA) (Ohkawa et al. 1979), reduced gluatathione levels (GSH) (Beutler et al. 1963) and superoxide dismutase levels (SOD) (Misra and Frodvich 1972) was performed.

\subsection{Mitochondrial DNA isolation and quantification}

Isolation of mitochondrial DNA (mtDNA) from the left ventricle of heart samples and mtDNA amount was determined by the method of Barja and Herrero (2000), by measuring the fluorescence after binding of the Hoechst 33258 dye.

\subsection{Histopathological and morphometric analysis}

Hematoxylin and Eosin staining of left ventricular tissue sections was carried out at Sukoon Pathologies Laboratory, Ahmedabad, Gujarat, India. Left ventricular tissue sections from 4 
hearts in each treatment were studied under OLYMPUS (trinocular-CX21FS1) microscope with $100 \mathrm{X}$ and $400 \mathrm{X}$ magnifications for examination of overall morphology of left ventricle and cell diameter measurements were taken with Image J analyzer software (NIH) 1.45.

\section{9 mRNA Expression Studies}

Total ribonucleic acid (RNA) was extracted from intact hearts using the FastRNA ${ }^{\circledR}$ Pro Green Kit (MPBIO) according to the manufacturer's instructions. The reverse transcription (RT) reaction was performed using First Strand cDNA Synthesis kit (Novagen). Real-time polymerase chain reaction (PCR) was performed in LightCycler ${ }^{\circledR} 480$ (Roche Applied Biosciences) using TaqMan Universal PCR Master mix for determining mRNA levels of histone deacetylase 2 (HDAC2) and histone deacetylase 5 (HDAC5). To detect HDAC 2 and HDAC 5, specific primers were designed with the 'Primer 3Plus' Program listed in table 1. All reactions were performed in duplicate. The PCR product was separated using electrophoresis on $1 \%$ agarose gel and semi-quantified as a ratio to GAPDH.

\subsection{Statistical Analysis:}

All the values were expressed as mean \pm S.E.M. Statistical analysis between normal control and disease control groups and between disease control and disease treated group were performed using one-way ANOVA followed by Tukey's posttest, using Prism 5.01 (GraphPad Software). Differences were considered to be statistically significant when $p<$ 0.05 .

\section{RESULTS}

\subsection{HYPERTROPHIC PARAMETERS}

PAAC model is a representative of pathological cardiac hypertrophic condition. To determine if PAAC induced cardiac hypertrophy could be treated with HDAC inhibition, we treated rats with magnesium valproate. It was observed that cardiac hypertrophic index (CHI), LV hypertrophy index (LVHI) was significantly higher in hypertrophic rats as compared to 
control rats. Treatment with magnesium valproate significantly reduced the elevated $\mathrm{CHI}$ and LVHI of hypertrophic rats (Figure 1A, 1B). Further, HW/BW and LVW/RVW were increased significantly in hypertrophic rats as compared to those of control rats. Magnesium valproate administration significantly reduced the increase in HW/BW and LVW/RVW of hypertrophic rats (Figure 1C, 1D). This was further confirmed by changes in LV wall thickness, cardiomyocyte diameter and LV collagen levels which was reduced by magnesium valproate treatment (Figure 2A, 2B, 2C).

\subsection{HEMODYNAMIC PARAMETERS}

In the earlier stage, hypertrophized ventricle is able to compensate for increased work load, but eventually in the later stages, impairment of diastolic function followed by systolic function is observed, resulting in decompensation and heart failure (Raghunathan and Patel 2013). Thus we evaluated the ventricular performance by invasive hemodynamic pressure recording and assessed the impact of HDACi magnesium valproate in cardiac functioning. As expected, there was a significant increase in the MABP and heart rate in PAAC hypertrophic rats as compared to control rats. Treatment with magnesium valproate significantly prevented the increase in MABP and attenuated increased heat rate in hypertrophic rats (Table 2).

Further, the hypertrophic rats also exhibited a significantly decreased rate of pressure development (dp/dt max) and decay (dp/dt min) as compared to control rats which was significantly increased by magnesium valproate treatment (Table 2 ).

\subsection{SERUM LIPID PROFILE}

Dyslipidaemia is one of the most modifiable risk factors for CVDs. PAAC is a model of pathological hypertrophy and is associated with decreased fatty acid oxidation (DavilaRoman et al. 2002). PAAC hypertrophic rats exhibited significantly increase in serum total cholesterol, LDL, VLDL and triglyceride levels and $\log$ TG/HDL ratio and decrease in serum HLD levels as compared to control rats. Treatment with magnesium valproate showed significant improvement in lipid profiles (Table 2). 


\subsection{NON-SPECIFIC SERUM CARDIAC MARKERS}

Concentrations of CK-MB have been found to be significantly higher in human myocardium with coronary artery disease, aortic stenosis, or heart failure (Hakan et al. 2002). A significant increased level of LDH is found in case of myocardial damage due to hypertrophy. CRP is a sensitive, nonspecific systemic marker of inflammation and its elevated levels are associated with traditional cardiovascular risk factors. Na-K-ATPase dysfunctioning has been found to be associated with myocardial contractile dysfunction. Hence, in present study, we have measured serum CK-MB, $\mathrm{LDH}$ and $\mathrm{CRP}$ levels as $\mathrm{LV} \mathrm{Na}+\mathrm{K}+$ ATPase activity. PAAC rats produced significant increase in serum LDH, CK-MB and CRP levels and reduction in LV $\mathrm{Na}^{+} \mathrm{K}^{+}$ATPase activity as compared to control rats. Treatment with magnesium valproate showed significant reduction in $\mathrm{LDH}, \mathrm{CK}-\mathrm{MB}$ and CRP levels and increase in $\mathrm{Na}^{+} \mathrm{K}^{+} \mathrm{ATPase}$ activity (Table 3).

\subsection{OXIDATIVE STRESS PARAMETERS}

Reactive oxygen species (ROS) aggravates cardiac hypertrophy in pressure overload induced cardiac hypertrophy with progression to heart failure (Han et al. 2009) and hence, measurement of pro-oxidants and anti-oxidant levels is suggestive of cardiac damage. In present investigation, hypertrophic control rats showed a significantly increase in LV MDA levels and significantly decreased LV SOD and GSH levels as compared to control rats. Treatment with magnesium valproate significantly decreased LV MDA levels and increased LV SOD and GSH of hypertrophic rats (Table 4).

\subsection{MITOCHONDRIAL DNA (mtDNA) CONCENTRATION}

Cardiac hypertrophy and heart failure have been frequently linked to accumulation of the deleted forms of mtDNA in the myocardium (Anan et al. 1995). Depletion of mtDNA and its reduced replication have been identified as markers of transition from compensated hypertrophy to right ventricular failure (Karamanlidis et al. 2011). Calf thymus DNA was used as standard DNA to construct the standard curve from which unknown concentrations of 
DNA samples were determined. A standard curve of total DNA vs. relative fluorescence unit (RFU) was plotted to generate the equation for calculation of mitochondrial DNA concentration where $\mathrm{r}^{2}$ was obtained as 0.9927 .

PAAC hypertrophic rats showed significantly reduced mitochondrial DNA (mtDNA) concentration as compared to control rats. Treatment with magnesium valporate significantly increased the reduced mtDNA concentration in hypertrophic treated rats as compared to hypertrophic control rats (Figure 3A).

\subsection{KAPLAN-MEIER PLOT FOR SURVIVAL PROBABILITY}

It is important to evaluate the long term efficacy and tolerability of magnesium valproate in clinical use. In lieu of this we monitored the animals for 6 weeks and plotted the KaplanMeier curve. Kaplan-Meier analysis revealed that the survival rate was 50\% at 35 days after surgery. However, administration of magnesium valproate improved the survival rate to $80 \%$ in PAAC model (Figure 3B).

\subsection{HISTOPATHOLOGICAL ANALYSIS}

Histopathological examination of longitudinal section of left ventricle (100X) in control and control treated with magnesium valproate rats showed normal long parallel cardiac fibres with normal interstitial space (Figure 4A, 4B). On the contrary, hypertrophic control rats revealed irregular cardiac fibres, reduction in interstitial space and fibrosis (Figure 4C, 4D) (100X).

Further, the histopathological analysis at $400 \mathrm{X}$ showed no signs of injury in control and control treated with magnesium valproate $(210 \mathrm{mg} / \mathrm{kg} /$ day, p.o.) (Figure $4 \mathrm{E}, 4 \mathrm{~F})$. However, hypertrophic control sections at $400 \mathrm{X}$ exhibited increased fibroblast, apoptotic cardiomyocyte, increased eosinophilia and extravasated RBCs (Figure 4G, 4H, 4I) as compared to control animals. Treatment with magnesium valproate in hypertrophic treated rats prevented the signs of hypertrophic damage to cardiomyocytes (Figure 4J).

\section{9 mRNA EXPRESSION OF HDAC2 AND HDAC5}


Class I HDAC enzymes have been implicated in promotion of cardiac hypertrophy through stimulation of pro-hypertrophic pathway (Kee and Kook 2011). Overexpression of HDAC 2 and its role in inhibition of anti-hypertrophic factors like KLF4 and Inpp5f has been found in ablation of cardiac hypertrophy (Kee and Kook 2011). On the contrary, class II HDAC enzymes have been implicated as inhibitors of pro-hypertrophic pathways. HDAC 5 has been found to interact with myocyte enhancer factor-2 (MEF2) and regress hypertrophy (Chang et al. 2004). These results would implicate the role of class IIa HDACs in prevention of cardiac hypertrophy and HDACi as promoters of cardiac hypertrophy. However, as mentioned earlier, experimental studies have revealed that non-specific HDACi suppress and block the progression of cardiac hypertrophy (Kook et al. 2003). In lieu of this, HDAC2, a class I HDAC enzyme and HDAC5, a class II HDAC mRNA levels were measured in the heart in present study.

Hypertrophic control rats exhibited a significant relative fold increase in HDAC 2 and HDAC 5 mRNA levels as compared to control rats. Treatment with magnesium valproate (210 $\mathrm{mg} / \mathrm{kg} /$ day, p.o.) significantly reduced the expression of HDAC 2 in hypertrophic rats as compared to control rats. However, administration of magnesium valproate in hypertrophic rats did not produce any significant change in HDAC 5 mRNA levels as compared to control rats (Figure 5A, 5B).

\section{DISCUSSION}

Results of the present investigation provide sufficient evidence that magnesium valproate prevents cardiac hypertrophy induced by PAAC, through inhibition of HDAC2. In the present investigation, HDAC2 and HDAC5 mRNA level were significantly increased in hypertrophic rats as compared to control rats. Chronic treatment with magnesium valproate significantly reduced the HDAC2 mRNA level, but had no significant effect on HDAC5 mRNA level. Further, valproic acid has been reported to have 5 times more potency for class I HDAC than 
class II HDAC (de Ruijter et al. 2003). Thus, magnesium valproate decreases expression of pro-hypertrophic HDAC i.e. HDAC2 without altering the expression of anti-hypertrophic HDAC5. This paradoxical involvement of both class IIa HDAC and HDACi simultaneously in prevention of cardiac hypertophy is attributed to three reasons-

1) Predomination of class I HDAC with regard to hypertrophic gene expression in heart (Kee et al. 2006).

2) Catalytic activity is not essential for class IIa HDACs to suppress hypertrophic signalling in cardiomyocytes (Zhang et al. 2002).

3) Enzymatic assays have revealed the insensitivity of standard HDACi (including those used in cardiac hypertophic studies till date) for class IIa HDACs (Bradner et al. 2010).

In the present study, magnesium valproate reduced hypertrophic indices and collagen levels in the treated rats. It has been reported that Hsp70-Hsp90 (Hop), heart enriched nuclear factor, is a cardiac specific regulator of gene transcription and generates hypertrophy by recruitment of Class I HDAC- specifically HDAC 2 (Kee and Kook 2011). It has also been suggested that in hypertrophic stress HDAC2 activity is regulated by HSP70 and the HSP70/HDAC2 axis plays an important role in inducing cardiac hypertophy (Kee and Kook 2011). Similarly Inpp5f, another HDAC2 downstream target has been found to regulate cardiac hypertrophy (Kee and Kook 2011). Inpp5f suppression due to HDAC2 overexpression activates the phosphatidylinositol 3-kinase (PI3K) Akt-Gsk3 $\beta$ pathway and leads to cardiac hypertrophy (Trivedi et al. 2007). Thus, magnesium valproate through its strong HDACi activity on class I HDAC might have prevented PAAC induced hypertrophy and collagen deposition.

Magnesium valproate also significantly improved the hemodynamics by reducing blood pressure, heart rate and improving left ventricular functioning. It is reported that Inpp5f suppression due to HDAC2 overexpression activates the phosphatidylinositol 3-kinase (PI3K) Akt-Gsk3 $\beta$ pathway, thus producing cardiac hypertrophy (Trivedi et al. 2007). This 
supports the possibility that improved hemodynamics and contractility by magnesium valproate in current study may be due to the modulation of (PI3K-) Akt-Gsk3 $\beta$ pathway by HDAC2 inhibition, which is a negative regulator of cardiac contractility.

In the present investigation, PAAC control rats exhibited significant dyslipidaemia. Chronic treatment with magnesium valproate significantly improved the lipid profile. It is reported that trichostatin A (TSA) treatment in F9 EC cells downregulated the expression levels of 9 out of 15 enzymes involved in cholesterol biosynthesis, including HMG CoA reductase, mevlonate kinase and others (Chittur et al. 2008). Further, reports suggest that valproic acid owing to its HDAC inhibitory activity blocked adipogenesis in mouse 3T3 L1 and human predipocytes (Lagace and Nachtigal 2004). Thus, control of dyslipidaemia by magnesium valproate indicates that magnesium valproate can prevent coronary artery diseases.

The results of present study suggest that there was a significant increase in serum CK-MB, $\mathrm{CRP}$ and $\mathrm{LDH}$ levels along with significant reduction of $\mathrm{Na}^{+} \mathrm{K}^{+}$ATPase activity in PAAC control rats. Treatment with magnesium valproate significantly reduced CK-MB, CRP and LDH levels, indicating a decrease in myocardial damage as well as reduction in cardiac hypertrophy. Further, chronic treatment with magnesium valproate in hypertrophic treated rats significantly restored the $\mathrm{Na}^{+} \mathrm{K}^{+}$ATPase activity. From these details it appears that magnesium valproate reduces levels of cardiac biomarkers, indicating prevention of cardiac damage and reduction in inflammation.

Treatment with magnesium valproate significantly prevented oxidative stress by reducing malondialdehyde levels and significantly increasing glutathione and superoxide dismutase levels. Further, hypertrophic control rats also exhibited decreased mtDNA concentration in left ventricle. Chronic treatment with magnesium valproate significantly increased the concentration of mtDNA. During pathological conditions such as heart failure, myocardial oxidative stress and mitochondrial dysfunctioning is aggravated by extra mitochondrial ROS sources (Ago et al. 2010; Kuroda et al. 2010). Further, reports suggest that oxidative stress 
leads to disruption of mitochondrial proteins and mtDNA mutation (Bugger and Abel 2010). It has been suggested that lack of complex chromatin organization consisting of histone proteins, limited the reparative ability of mtDNA and impermeability of internally formed $\mathrm{O}^{2-}$ in mitochondria, may be the possible reasons for ROS mediated mtDNA damage (Ide et al. 2001). It has been reported that owing to its HDACi activity, phenylbutyrate might have increased MnSOD levels against adriamycin toxicity in a mouse model (Daosukho et al. 2007). It has been shown that localized deacetylation of H3 and H4 in MnSOD gene's proximal promoter region which is executed by HDAC1, was reversed by class I specific HDACi- trichostatin A (Maehara et al. 2002). In another study, HDACi by valproic acid attenuated the increased ROS in spontaneously hypertensive rats (Cardinale et al. 2010). Thus, magnesium valproate, through its HDACi activity prevented oxidative stress and thereby preserved cardiovascular function by preventing reduction in mtDNA concentration in hypertrophic treated animals.

Improvement in cardiac hypertrophy was further evident by histopathological study of the transverse section of left ventricle tissue. Histopathological examination in PAAC control rats revealed reduction of interstitial space, enlarged cardiomyocyte, increased eosinophilia, extravasated RBCs and apoptosis. Treatment with magnesium valproate in hypertrophic treated rats showed less reduction in interstitial space, less increase in cardiomyocyte diameter and reduction in eosinophilia, extravasation of RBCs and apoptosis as compared to hypertrophic control rats. Intense fibrosis was observed in LV section of the hypertrophic rat. Treatment with magnesium valproate in hypertrophic rats showed reduced fibrosis in LV section indicative of beneficial effect of magnesium valproate. Further, Kaplan-Meier curve indicated that administration of magnesium valproate improved the survival rate in hypertrophic treated rats as compared to hypertrophic control rats.

This is a molecular pharmacological investigation which provides an insight into newer targets for cardiac hypertrophy suggesting that selective class I HDAC inhibition is required 
for controlling cardiac hypertrophy. This study can be further expanded by developing new chemical entities by modifying the structure of magnesium valproate. Focus should be laid along the lines of achieving specificity and selectivity for particular class of HDAC enzymes i.e. their major action on class I over class II HDAC. This would ensure curbing the disease at core, eliminating side-effects and associated complications, which are major drawback for existing therapies.

\section{CONCLUSION}

In conclusion, this study gives an insight that selective class I HDACi are required for controlling cardiac hypertrophy. Till date, the HDACi used are non-selective, differing in terms of relative selectivity. Hence, this study is one of its kind suggesting that modifying the structure of magnesium valproate to obtain still more selectivity towards class I HDAC should be a strategy. Furthermore, newer HDAC inhibitors which are class I inhibitor and class II promoter can be designed to obtain a 'pan' or 'dual' natural HDAC 'regulators'.

Conflict of Interest: None declared.

\section{REFERENCES:}

Ago, T., Kuroda, J., Pain, J., Fu, C., Li, H., and Sadoshima, J. 2010. Upregulation of Nox4 by hypertrophic stimuli promotes apoptosis and mitochondrial dysfunction in cardiac myocytes. Circ. Res. 106:1253-1264.

Anan, R., Nakagawa, M., Miyata, M., Higuchi, I., Nakao, S., Suehara, M., et al. 1995. Cardiac involvement in mitochondrial diseases: a study on 17 patients with documented mitochondrial DNA defects. Circulation, 91:955-961.

Barja, G., and Herrero, A. 2000 Oxidative damage to mitochondrial DNA is inversely related to maximum life span in the heat and brain of mammals. FASEB J. 14:312-318. 
Beutler, E., Duron, O., and Kelly, B. 1962. Improved method for the determination of blood glutathione. J. Lab. Clin. Med. 61: 882-888.

Bradner, J.E., West, N., Grachan, M.L., Greenberg, E.F., Haggarty, S.J., Warnow, T., et al, 2010. Chemical phylogenetics of histone deacetylases. Nat. Chem. Biol. 6:238-243.

Bugger, H., and Abel, E.D. 2010. Mitochondria in the diabetic heart. Cardiovasc. Res. 88: 229-240.

Cardinale, J.P., Sriramula, S., Pariaut, R., Guggilam, A., Mariappan, N., Elks, C.M., et al. 2010. HDAC inhibition attenuates inflammatory, hypertrophic, and hypertensive responses in spontaneously hypertensive rats. Hypertension, 56:437-444.

Chang, S., McKinsey, T.A., Zhang, C.L., Richardson, J.A., Hill, J.A., and Olson, E.N. 2004. Histone deacetylases 5 and 9 govern responsiveness of the heart to a subset of stress signals and play redundant roles in heart development. Mol. Cell. Biol. 24:8467-8476.

Chittur, S.V., Sangster-Gity, N., and McCormick, P.J. 2008. Histone deactylase inhibitors: A new mode for inhibition of cholesterol metabolism. BMC Genomics, 9:507.

Daosukho, C., Chen, Y., Noel, T., Sompol, P., Nithipongvanitch, R., Velez, J.M., et al, 2007. Phenylbutyrate, a histone deacetylase inhibitor, protects against Adriamycin-induced cardiac injury. Free Radic. Biol. Med. 42:1818-1825.

Davila-Roman, V.G., Vedala, G., Herrero, P., de las Fuentes, L., Rogers, J.G., Kelly, D.P., et al. 2002. Altered myocardial fatty acid and glucose metabolism in idiopathic dilated cardiomyopathy. J. Am. Coll. Cardiol. 40:271-277.

de Ruijter, A.J.M., van Gennip, A.H., Caron, H.N., Kemp, S., and van Kuilenburg, A.B.P. 2003. Histone deacetylases (HDACs): characterization of the classical HDAC family. Biochem. J. 370:737-749.

Frey, N., Katus, H.A., Olson, E.N., and Hill, J.A. 2004. Hypertrophy of the Heart: A New Therapeutic Target? Circulation, 109:1580-1589. 
Goyal, B.R., and Mehta, A.A. 2012. Beneficial role of spironolactone, telmisartan and their combination on isoproterenol induced cardiac hypertrophy. Acta Cardiol. 67:203-211.

Goyal, B.R., and Mehta, A.A. 2013. Diabetic cardiomyopathy: Pathophysiological mechanisms and cardiac dysfunction. Hum. Exp. Toxicol. 32: 571-590.

Goyal, B.R., Mesariya, P., Goyal, R.K., and Mehta, A.A. 2008. Effect of telmisartan on cardiovascular complications associated with streptozotocin diabetic rats. Mol. Cell. Biochem. 314:123-131.

Goyal, B.R., Parmar, K., Goyal, R.K., and Mehta, A.A. 2011. Beneficial role of telmisartan on cardiovascular complications associated with STZ-induced type-2 diabetic rats. Pharmacol. Rep. 63:956-966.

Goyal, B.R,, Patel, M.M., and Bhadada, S.V. 2011. Comparative evaluation of spironolactone, atenolol, metoprolol, ramipril and perindopril on diabetes induced cardiovascular complications in type 1 diabetes in rats. Int. J. Diabetes Metab. 19:11-18.

Goyal, B.R., Solanki, N., Goyal, R.K., and Mehta, A.A. 2009. Investigation into the cardiac effects of spironolactone in the experimental model of type 1 diabetes. J. Cardiovasc. Pharmacol. 54:502-509.

Gregoretti, I.V., Lee, Y.M., and Goodson, H.V. 2004. Molecular evolution of the histone deacetylase family: functional implications of phylogenetic analysis. J. Mol. Biol. 338:1731.

Hakan, A.Y., Arsava, M., Okay, S. 2002. Creatine Kinase-MB Elevation After Stroke Is Not Cardiac in Origin. Stroke, 33:286-290.

Han, J.J., Hao, J., Kim, C.H., Hong, J.S., Ahn, H.Y., and Lee, Y.S. 2009. Quercetin Prevents Cardiac Hypertrophy Induced by Pressure Overload in Rats. J. Vet. Med. Sci. 71:737-43.

Ide, T., Tsutsui, H., Hayashidani, S., Kang, D., Suematsu, N., Nakamura, K., et el. 2001. Mitocondrial DNA damage and dysfunction associated with oxidative stress in failing hearts after myocardial infarction. Circ. Res. 88:529-535. 
Karamanlidis, G., Bautista-Hernandez, V., Fynn-Thompson, F., Del Nido, P., and Tian, R. 2011. Impaired mitochondrial biogenesis precedes heart failure in right ventricular hypertrophy in congenital heart disease. Circ. Heart Fail. 4:707-13.

Kee, H.J., and Kook, H. 2011. Roles and Targets of Class I and IIa Histone Deacetylases in Cardiac Hypertrophy. J. Biomed. Biotechnol. 2011::928326. Epub 2010.

Kee, H.J., Sohn, I.S., Nam, K.I., Park, J.E., Qian, Y.R., Yin, Z., et al. 2006. Inhibition of Histone Deacetylation Blocks Cardiac Hypertrophy Induced by Angiotensin II Infusion and Aortic Banding. Circulation, 113:51-59.

Kook, H., Lepore, J.J., Gitler, A.D., Lu, M.M., Wing-Man Yung, W., Mackay, J., et al. 2003. Cardiac hypertrophy and histone deacetylase-dependent transcriptional repression mediated by the atypical homeodomain protein Hop. J. Clin. Invest. 112:863-871.

Kuroda, J., Ago, T., Matsushima, S., Zhai, P., Schneider, M.D., and Sadoshima, J. 2010. NADPH oxidase 4 (Nox4) is a major source of oxidative stress in the failing heart. Proc. Natl. Acad. Sci. U.S.A. 107:15565-15570.

Lagace, D.C., and Nachtigal, M.W. 2004. Inhibition of Histone Deacetylase Activity by Valproic Acid. J. Biol. Chem. 279:18851-18860.

Lowry, O.H., Rosenbrough, N.J., Farr, A.L., and Randall, R.J. 1951. Protein measurement with folin phenol reagent. J. Biol. Chem. 193:265-275.

Maehara, K., Uekawa, N., and Isobe, K.I. 2002. Effects of histone acetylation on transcriptional regulation of manganese superoxide dismutase gene. Biochem. Biophys. Res. Commun. 295:187-192.

Misra, H.P., and Frodvich, I. 1972. The role of superoxide anion in the autoxidation of epinephrine and a simple assay for superoxide dismutase. J. Biol. Chem. 247:3170-3175.

Ohkawa, H., Ohishi, N., and Yagi, K. 1979. Assay for lipid peroxides in animal tissue by thiobarbituric acid reaction. Anal. Biochem. 95:351-358. 
Patel, B.M., and Mehta, A.A. 2013. Choice of antihypertensive agents in diabetes subjects. Diab. Vasc. Dis. Res. 10:385-396.

Patel, B.M., Agarwal, S.S., and Bhadada, S.V. 2012. Perindopril protects against streptozotocin-induced hyperglycemic myocardial damage/alterations. Hum. Exp. Toxicol. 31:1138-1149.

Patel, B.M., and Bhadada, S.V. 2014. Type 2 diabetes induced cardiovascular complications: Comparative evaluation of spironolactone, atenolol, metoprolol, ramipril and perindopril. Clin. Exp. Hypertens. 36(5): 340-347

Patel, B.M., and Desai, V.J. 2014. Beneficial role of tamoxifen in experimentally induced cardiac hypertrophy. Pharmacol. Rep. 66(2): 264-272

Patel, B.M., Kakadiya, J., Goyal, R.K., and Mehta, A.A. 2013. Effect of spironolactone on cardiovascular complications associated with type-2 diabetes in rats. Exp. Clin. Endocrinol. 121: $441-447$

Patel, B.M., and Mehta, A.A. 2012. Aldosterone and angiotensin: Role in diabetes and cardiovascular diseases. Eur. J. Pharmacol. 697:1-12.

Patel, B.M., Raghunathan, S., and Porwal, U. 2014. Cardioprotective effects of magnesium valproate in type 2 diabetes mellitus. Eur. J. Pharmacol. 728: 128-134

Raghunathan, S., and Patel, B.M. 2013. Therapeutic implications of small interfering RNA in cardiovascular diseases. Fundam. Clin. Pharmacol. 27:1-20.

Raghunathan, S., Tank, P., Bhadada, S.V., and Patel, B.M. 2014. Evaluation of Buspirone on Streptozotocin induced Type 1 Diabetes and its associated complications. BioMed Res. Int. 2014: 9 pages Article ID 948427 (doi: 10.1155/2014/948427)

Rayabarapu, N., and Patel, B.M. 2014. Beneficial role of tamoxifen in isoproterenol induced myocardial infarction. Can. J. Physiol. Pharmacol. 92(10): 849-857 
Trivedi, C.M., Luo, Y., Yin, Z., Zhang, M., Zhu, W., Wang, T., et al. 2007. Hdac2 regulates the cardiac hypertrophic response by modulating Gsk3 $\beta$ activity. Nat. Med. 13:324-331.

Zhang, C.L., McKinsey, T.A., Chang, S., Antos, C.L., Hill, J.A., and Olson, E.N.2002. Class II histone deacetylases act as signal-responsive repressors of cardiac hypertrophy. Cell, 110:479-488. 
TABLE 1: Sequence of the primers for RT-PCR

\begin{tabular}{lllc}
\hline mRNA & SENSE PRIMER & ANTISENSE PRIMER & $\begin{array}{c}\text { Product } \\
\text { sizes (bp) }\end{array}$ \\
\hline HDAC2 & 5'-ACTTGCCGTTGCTGATGCTT-3' & 5'-TTGAACACCAGGCGCATGT-3' & 266 \\
\hline HDAC5 & 5'-GCAGGAGAGCTCAAGAATGGA-3' & 5'-AAGTTCCCATTGTCGTAGCGA-3' & 251 \\
\hline
\end{tabular}

HDAC 2- Histone deacetyalse 2

HDAC 5- Histone deacetyalse 5 
TABLE 2. Effect of Magnesium valproate on Blood pressure, Heart rate and lipid profile

\begin{tabular}{lcccc}
\hline PARAMETERS & CON & COM & DIS & DIM \\
\hline Blood Pressure (mmHg) & $122.6 \pm 2.94$ & $119.18 \pm 0.97$ & $154.4 \pm 3.63^{*}$ & $126.67 \pm 2.48^{* *}$ \\
\hline Heart Rate (beats/min) & $238 \pm 8.97$ & $295 \pm 4.61$ & $368 \pm 10.07^{*}$ & $278 \pm 6.64^{* *}$ \\
\hline Total Serum Cholesterol & $81.67 \pm 3.41$ & $79.91 \pm 3.27$ & $128.19 \pm 6.85^{*}$ & $92.34 \pm 1.87^{* *}$ \\
(mg/dl) & & & & \\
\hline Serum LDL (mg/dl) & $37.41 \pm 5.83$ & $37.33 \pm 3.44$ & $87.21 \pm 7.49^{*}$ & $44.82 \pm 3.43^{* *}$ \\
& & & & $17.67 \pm 0.64^{*}$ \\
\hline Serum VLDL (mg/dl) & $9.77 \pm 0.54$ & $9.43 \pm 0.65$ & $12.90 \pm 1.49^{* *}$ \\
\hline Serum TG (mg/dl) & $48.87 \pm 2.71$ & $47.1 \pm 3.23$ & $88.34 \pm 3.18^{*}$ & $64.54 \pm 7.47^{* *}$ \\
\hline Serum HDL (mg/dl) & $34.48 \pm 2.31$ & $33.14 \pm 1.97$ & $23.31 \pm 1.97^{*}$ & $34.61 \pm 1.56^{* *}$ \\
\hline logTG/HDL ratio & $0.049 \pm 0.0027$ & $0.051 \pm 0.0033$ & $0.085 \pm 0.0076^{*}$ & $0.052 \pm 0.0015^{* *}$ \\
\hline
\end{tabular}

* Significantly different from normal control group $(p<0.05)$.

** Significantly different from hypertrophic control group $(p<0.05)$.

CON -Sham Control

$\mathrm{COM}$-Sham control animals treated with magnesium valproate $(210 \mathrm{mg} / \mathrm{kg} /$ day, p.o.)

DIS - Hypertrophic control animals

DIM - Hypertrophic animals treated with magnesium valproate $(210 \mathrm{mg} / \mathrm{kg} / \mathrm{day}$, p.o. $)$ 
TABLE 3. Effect of Magnesium valproate on non-specific cardiac biomarkers

\begin{tabular}{lcccc}
\hline PARAMETERS & CON & COM & DIS & DIM \\
\hline Lactate Dehydrogenase & $653.30 \pm 6.67$ & $713.80 \pm 35.63$ & $1409.58 \pm 53.56^{*}$ & $904.31 \pm 7.85^{* *}$ \\
$(\mathrm{U} / \mathrm{l})$ & & & & \\
\hline Creatinine Kinase-MB & $491.80 \pm 16.53$ & $564.16 \pm 38.71$ & $825.85 \pm 30.90^{*}$ & $567.82 \pm 58.39^{* *}$ \\
$(\mathrm{U} / \mathrm{l})$ & & & & \\
\hline C-Reactive Protein & $7.49 \pm 0.74$ & $7.31 \pm 0.49$ & $19.53 \pm 0.38^{*}$ & $14.07 \pm 0.41^{* *}$ \\
$(\mathrm{mg} / \mathrm{l})$ & & & & \\
\hline Na ${ }^{+} \mathrm{K}^{+}$ATPase & & & & \\
ACTIVITY(nmoles of Pi & $16.64 \pm 2.19$ & $14.89 \pm 1.69$ & $5.40 \pm 0.54^{*}$ & $11.27 \pm 1.01^{* *}$ \\
liberated $/ \mathrm{hr} / \mathrm{mg}$ protein) & & & & \\
\hline
\end{tabular}

* Significantly different from normal control group $(p<0.05)$.

** Significantly different from hypertrophic control group $(p<0.05)$.

CON -Sham Control

COM -Sham control animals treated with magnesium valproate $(210 \mathrm{mg} / \mathrm{kg} /$ day, p.o.)

DIS - Hypertrophic control animals

DIM - Hypertrophic animals treated with magnesium valproate $(210 \mathrm{mg} / \mathrm{kg} /$ day, p.o.) 
TABLE 4. Effect of Magnesium valproate on left ventricular oxidative stress markers

\begin{tabular}{lcccc}
\hline PARAMETERS & CON & COM & DIS & DIM \\
\hline LV Malondialdehyde level & $2.53 \pm 0.17$ & $3.23 \pm 0.31$ & $6.54 \pm 0.83^{*}$ & $4.22 \pm 0.25^{* *}$ \\
$(\mathrm{nmol} / \mathrm{mg}$ protein) & & & & \\
\hline LV Superoxide dismutase & $3.41 \pm 0.32$ & $3.73 \pm 0.24$ & $1.13 \pm 0.35^{*}$ & $2.86 \pm 0.07^{* *}$ \\
level (unit/min/mg protein) & & & & \\
\hline LV Glutathione level & $3.69 \pm 0.41$ & $4.23 \pm 0.58$ & $1.68 \pm 0.18^{*}$ & $3.30 \pm 0.18^{* *}$ \\
$(\mu \mathrm{g} / \mathrm{mg}$ protein) & & & & \\
\hline
\end{tabular}

* Significantly different from normal control group $(p<0.05)$.

** Significantly different from hypertrophic control group $(p<0.05)$.

CON -Sham Control

COM -Sham control animals treated with magnesium valproate $(210 \mathrm{mg} / \mathrm{kg} /$ day, p.o.)

DIS - Hypertrophic control animals

DIM - Hypertrophic animals treated with magnesium valproate $(210 \mathrm{mg} / \mathrm{kg} / \mathrm{day}$, p.o.) 


\section{Figure Legends}

Figure 1: Effect of Magnesium valproate on change in (A) cardiac hypertrophic index (CHI) (B) Left ventricular hypertrophic index (LVHI) (C) Heart weight to body weight ratio (HW/BW) (D) Left ventricular weight to heart weight ratio $(\mathrm{LVW} / \mathrm{HW}) .{ }^{*}$ significantly different from normal control $(p<0.05) .{ }^{* *}$ significantly different from hypertrophic control $(p<0.05)$. Each bar represents Mean \pm SEM of 6 rats. CON - Normal control. COB - Control treated with magnesium valproate, DIS Hypertrophic control. DIM - Hypertrophic treated with magnesium valproate.

Figure 2: Effect of Magnesium valproate on (A) Left ventricular wall thickness (B) cardiomyocyte diameter (C) Left ventricular collagen levels. * significantly different from normal control $(p<0.05)$. ** significantly different from hypertrophic control $(p<0.05)$. Each bar represents Mean \pm SEM of 6 rats. CON - Normal control. COB - Control treated with magnesium valproate, DIS - Hypertrophic control. DIM - Hypertrophic treated with magnesium valproate.

Figure 3: Effect of Magnesium valproate on change in (A) Mitochondrial DNA concentration (B) Kaplan Meir analysis. * significantly different from normal control $(p<0.05)$. ** significantly different from hypertrophic control $(p<0.05)$. Each bar represents Mean \pm SEM of 6 rats. CON Normal control. COB - Control treated with magnesium valproate, DIS - Hypertrophic control. DIM - Hypertrophic treated with magnesium valproate.

Figure 4: Representative figures of cardiac fibres at 100X: (A) Control, (B) Control treated, Showing normal long parallel longitudinal cardiomyocytes with normal interstitial space (i). (C) PAAC hypertrophic control: Irregular cardiac fibres, reduced interstitial space (Ri) and fibrosis (f), (D) PAAC hypertrophic treated: organized cardiac fibres, less reduction in interstitial space (ri), reduced fibrosis (rf). At 400X: (E) Control, (F) Control treated: Showing normal long parallel longitudinal cardiomyocytes, with no apoptosis, fibrosis or extravasated RBCs. (G), (H), (I) PAAC hypertrophic control: increased fibroblasts (f), apoptotic cardiomyocyte (a), increased eosinophilia (e), extravasated RBCs (R). (J) PAAC hypertophic treated: absence of apoptosis, extravasated RBC and eosinophilia.

Figure 5: Effect of Magnesium valproate on change in (A) HDAC 2 mRNA levels (B) HDAC 5 mRNA levels. * significantly different from normal control $(p<0.05) .{ }^{* *}$ significantly different from 
hypertrophic control $(p<0.05)$. Each bar represents Mean \pm SEM of 6 rats. CON - Normal control. COB - Control treated with magnesium valproate, DIS - Hypertrophic control. DIM - Hypertrophic treated with magnesium valproate. 

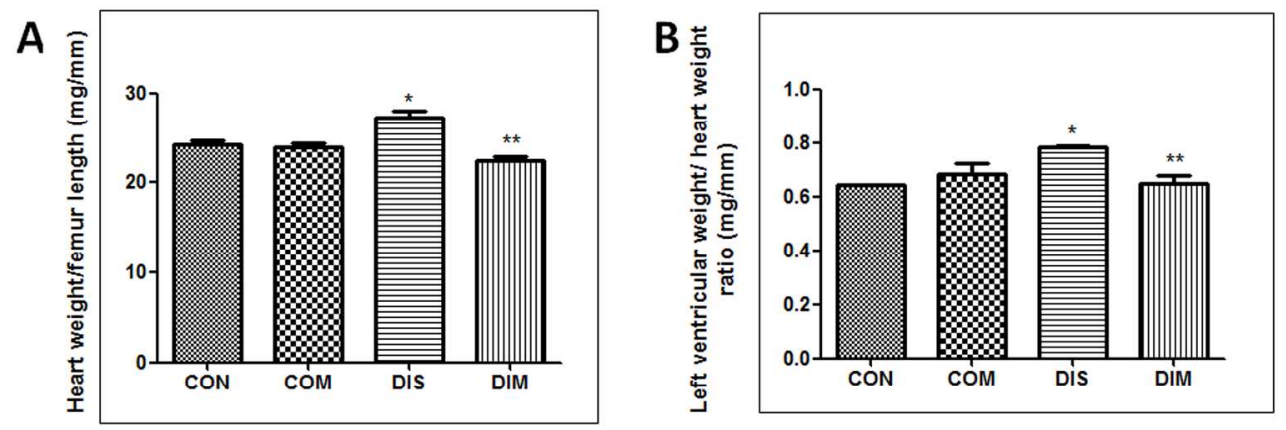

C

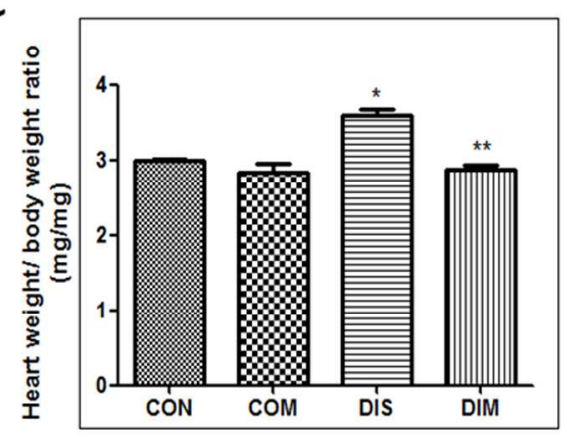

D

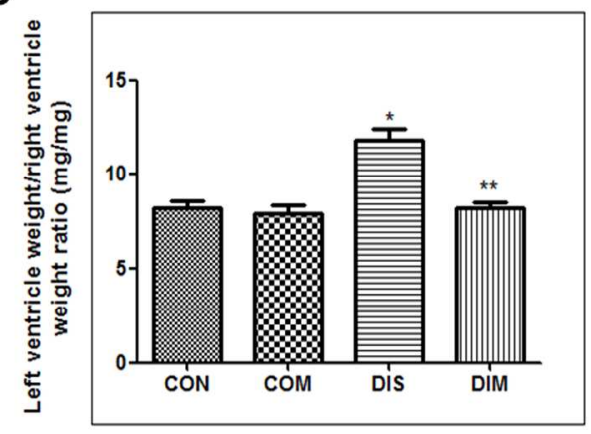

Figure 1.

$341 \times 241 \mathrm{~mm}(300 \times 300$ DPI $)$ 

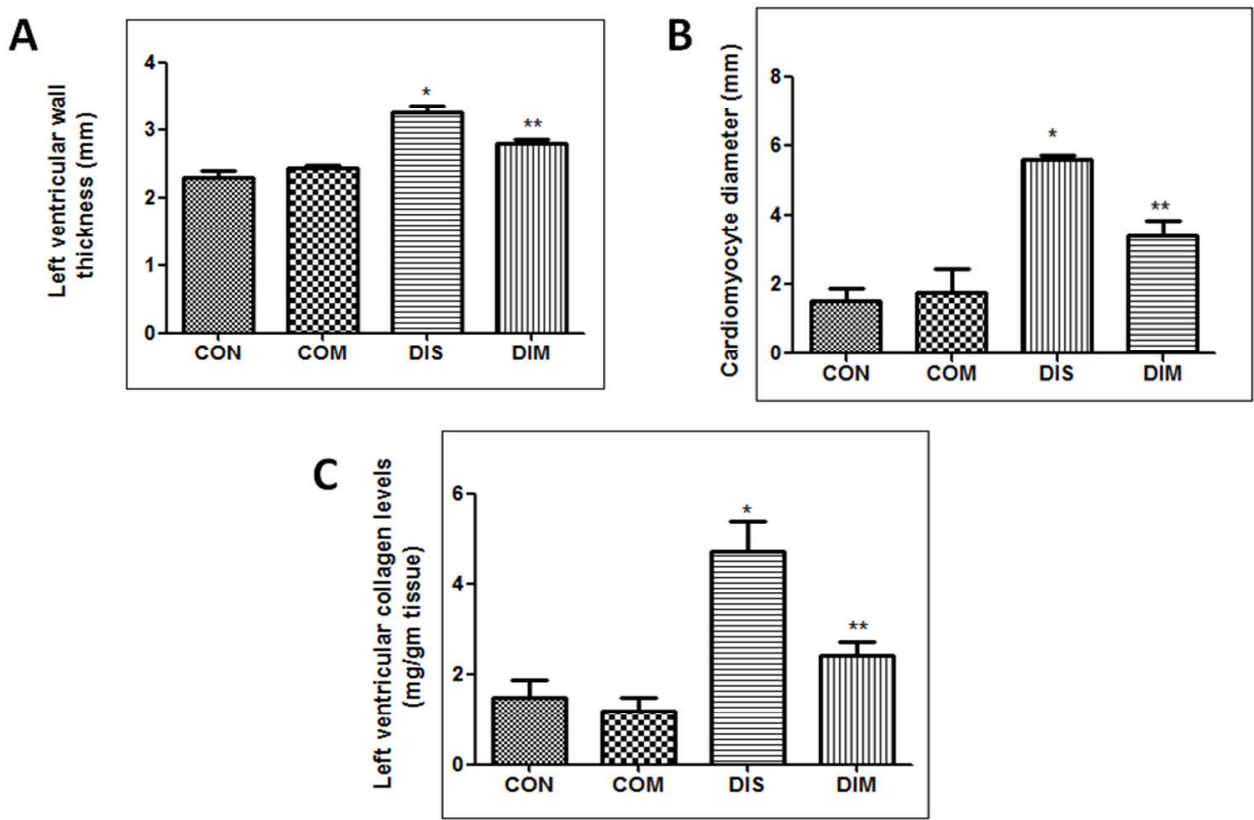

Figure 2.

$339 \times 220 \mathrm{~mm}(300 \times 300 \mathrm{DPI})$ 

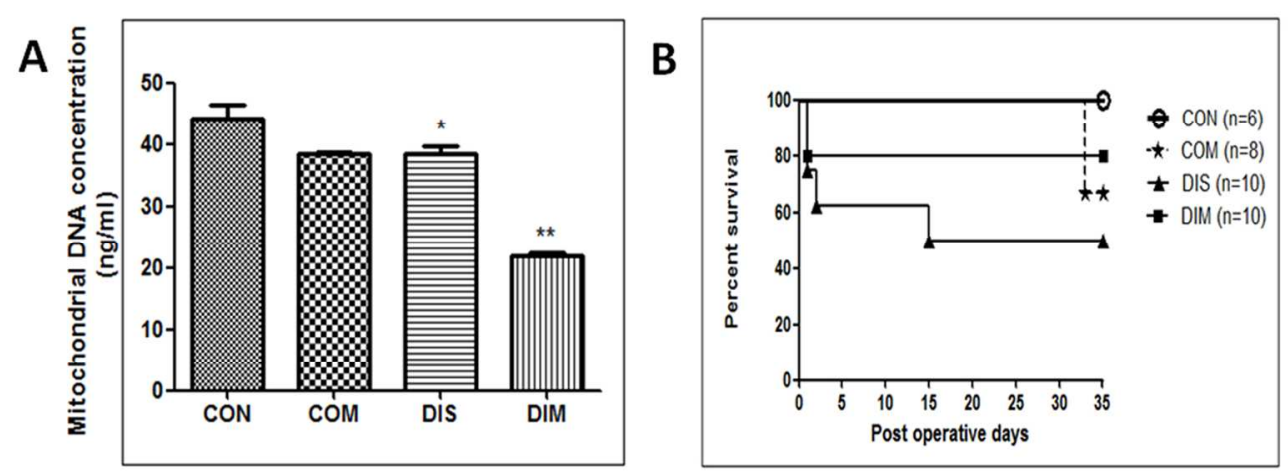

Figure 3.

$124 \times 45 \mathrm{~mm}(300 \times 300 \mathrm{DPI})$ 

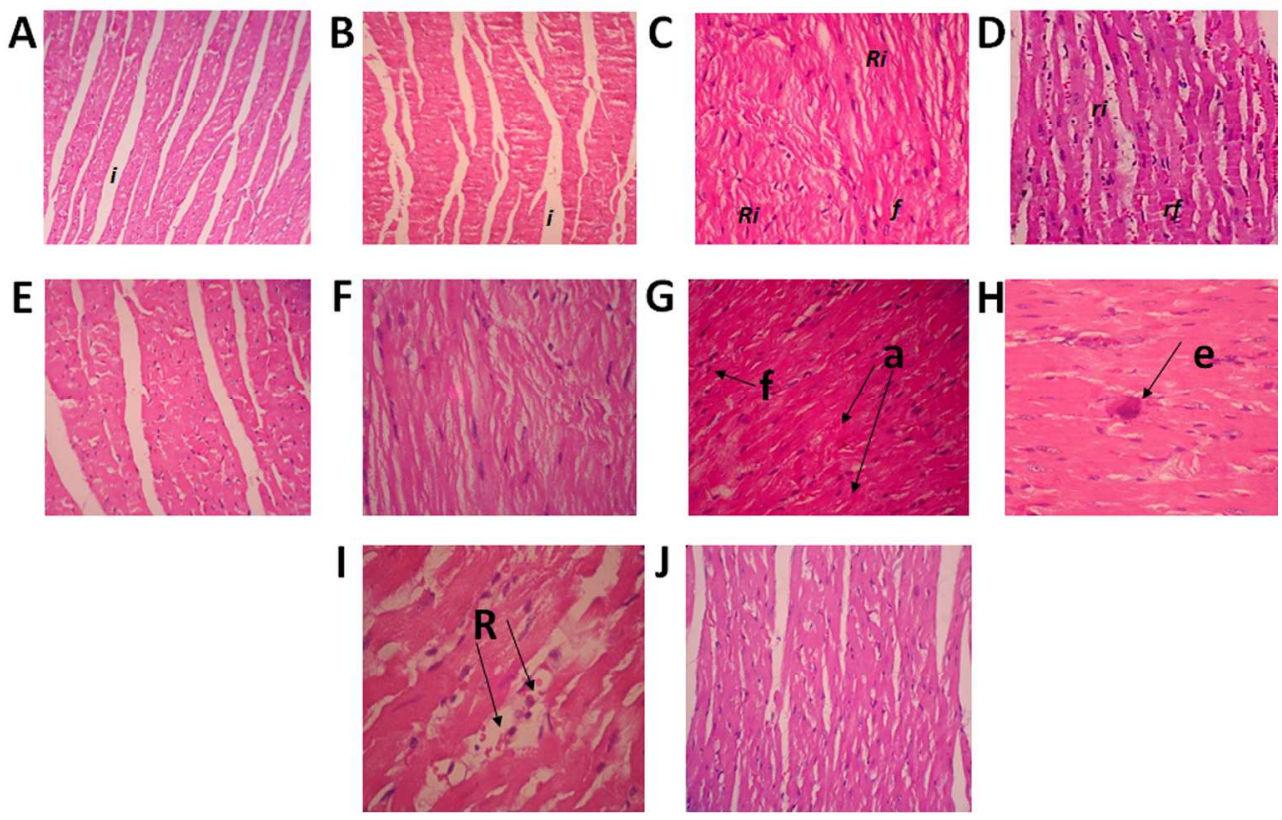

Figure 4.

$345 \times 219 \mathrm{~mm}(300 \times 300 \mathrm{DPI})$ 

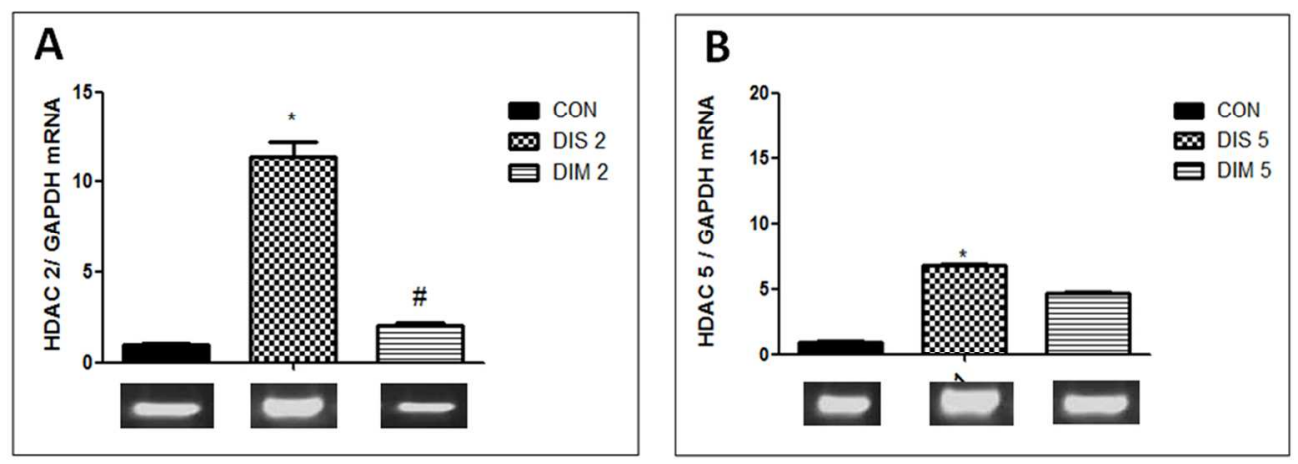

Figure 5.

$122 \times 44 \mathrm{~mm}(300 \times 300 \mathrm{DPI})$ 